\title{
Interfacial Proton Transfer for Hydrogen Evolution at the Sub- Nanometric Platinum/Electrolyte Interface
}

Hao Zhang ${ }^{1,2}$, Lina $\mathrm{Cao}^{3 \star}$, Yanlei Wang ${ }^{4}$, Zhongdong Gan ${ }^{4}$, Fanfei Sun ${ }^{5}$, Meiling Xiao6, Yuqi Yang ${ }^{1,7}$, Bingbao Mei ${ }^{1,7}$, Dongshuang $\mathrm{Wu}^{8 *}$, Junling $\mathrm{Lu}^{3}$, Hongyan $\mathrm{He}^{4 *}$, Zheng Jiang ${ }^{1,4,5 *}$

${ }^{1}$ Shanghai Institute of Applied Physics, Chinese Academy of Sciences, Shanghai 201800, China

2Institute of Functional Nano and Soft Materials Laboratory (FUNSOM), Jiangsu Key Laboratory for Carbon-Based Functional Materials \& Devices Soochow University, Suzhou 215123, China

${ }^{3}$ Hefei National Laboratory for Physical Sciences at the Microscale, University of Science and Technology of China, Hefei 230026, China

${ }^{4}$ Beijing Key Laboratory of Ionic Liquid Clean Process, CAS Key Laboratory of Green Process and Engineering, Institute of Process Engineering, Chinese Academy of Sciences, Bejing 100190, China.

${ }^{5}$ Shanghai Synchrotron Radiation Facility, Zhangjiang National Lab, Shanghai Advanced Research Institute, Chinese Academy of Sciences, Shanghai 201204, China ${ }^{6}$ State Key Laboratory of Electroanalytical Chemistry, Changchun Institute of Applied Chemistry, Chinese Academy of Sciences, Changchun 130022, China

${ }^{7}$ University of Chinese Academy of Sciences, Beijing 100049, China

${ }^{8}$ Division of Chemistry, Graduate School of Science, Kyoto University, Kitashirakawa Oiwakechho, Sakyoku, Kyoto 606-8502, Japan

*Authors contributed equally to this work.

E-mail: jiangzheng@sinap.ac.cn; $\quad$ Dongshuangwu@kuchem.kyoto-u.ac.jp; hyhe@,ipe.ac.cn 


\section{Contents}

\section{Suppleme ntary Figures}

Figure S1. HAADF-STEM images of SAC-Pt and SNM-Pt.

Figure S2. WT-plots of SNM-Pt, SAC-Pt, Pt foil and $\mathrm{PtO}_{2}$.

Figure S3. Equipment used for operando XAS measurements.

Figure S4. The raw $k^{2}$-weighted k-space spectra of SNM-Pt at different potentials

Figure S5. The XAS spectra evolution of SNM-Pt and SAC-Pt after the electrode preparation on $\mathrm{CC}$.

Figure S6. Operando XANES spectra of SAC-Pt at different potentials.

Figure S7. Spectra showing the relationship between the integrated white-line area and formal 5d-band hole count.

Figure S8. Operando FT-EXAFS spectra of SAC-Pt at different potentials.

Figure S9-S16. Experimental and fitted EXAFS curves in both R and $\mathrm{k}$ space for SNM-Pt at different potentials.

\section{Suppleme ntary Tables}

Table S1. Comparison of HER performance between SNM-Pt and the recently reported Pt-based electrocatalysts.

Table S2. Quantitative FT-EXAFS curves fitting parameters for SNM-Pt.

Table S3. The average Pt-Pt CN calculated from the optimized models.

\section{References}




\section{Supplementary Figures}
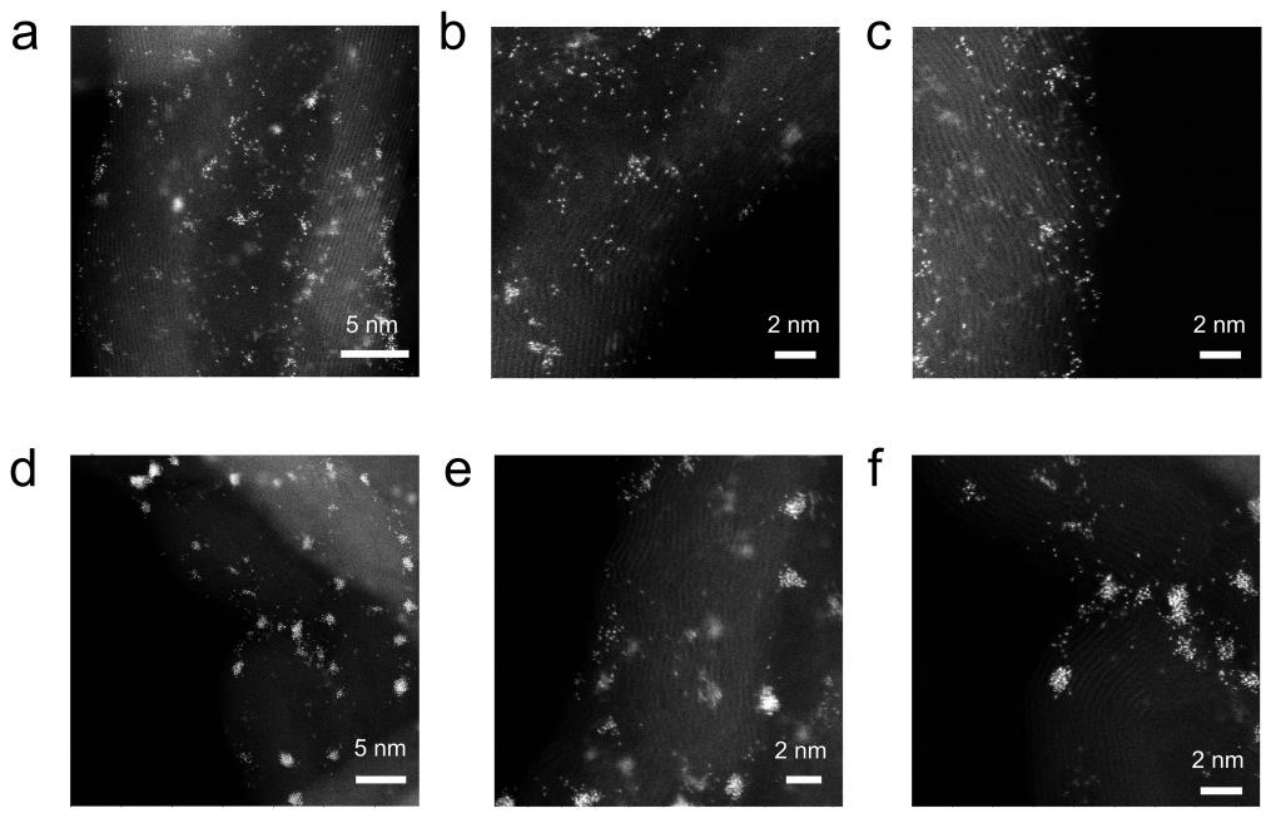

Figure S1. HAADF-STEM images of SAC-Pt (a-c) and SNM-Pt (d-f). 

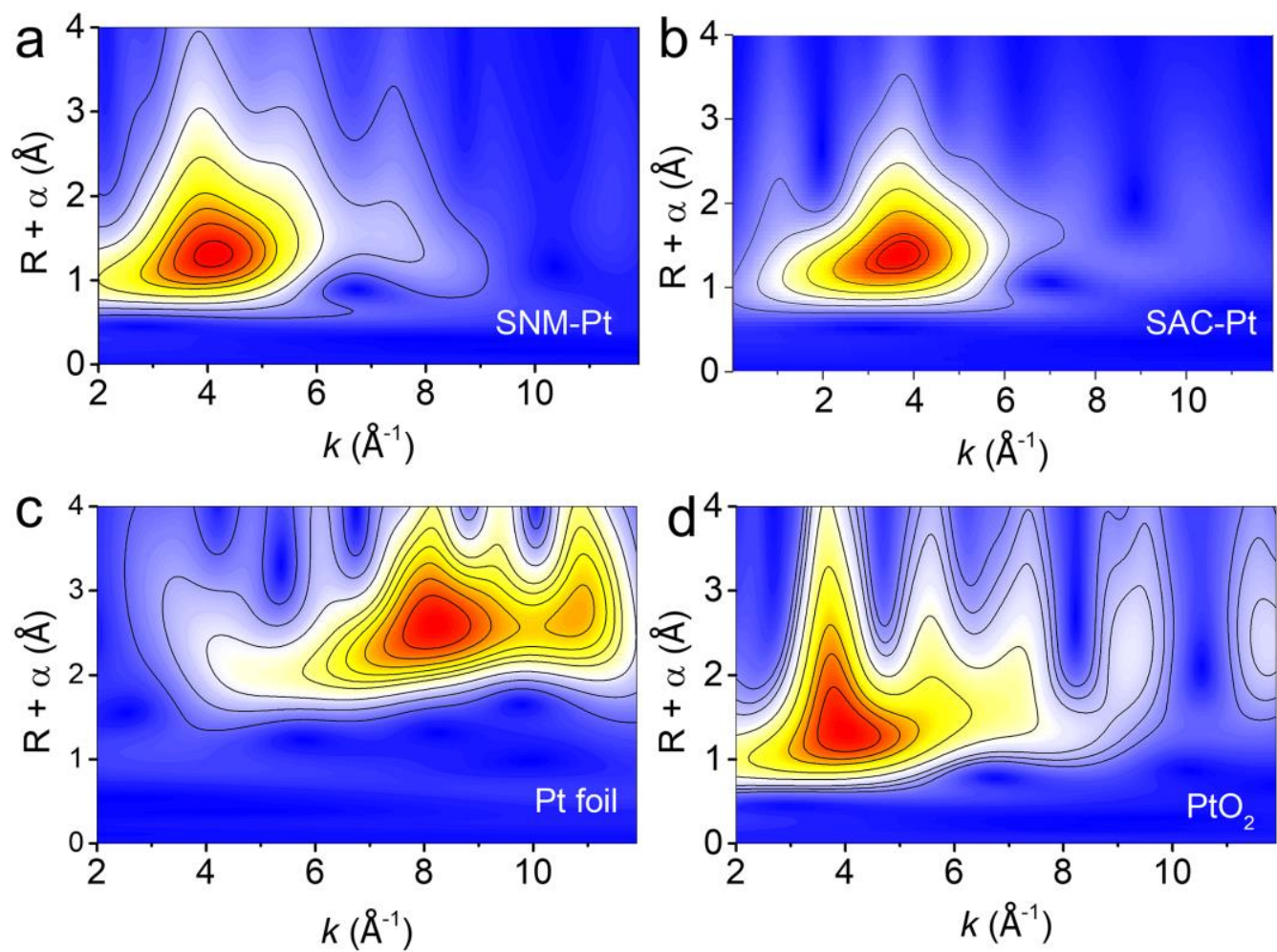

Figure S2. WT-plots of (a) SNM-Pt, (b) SAC-Pt, (c) Pt foil and (d) $\mathrm{PtO}_{2}$. 


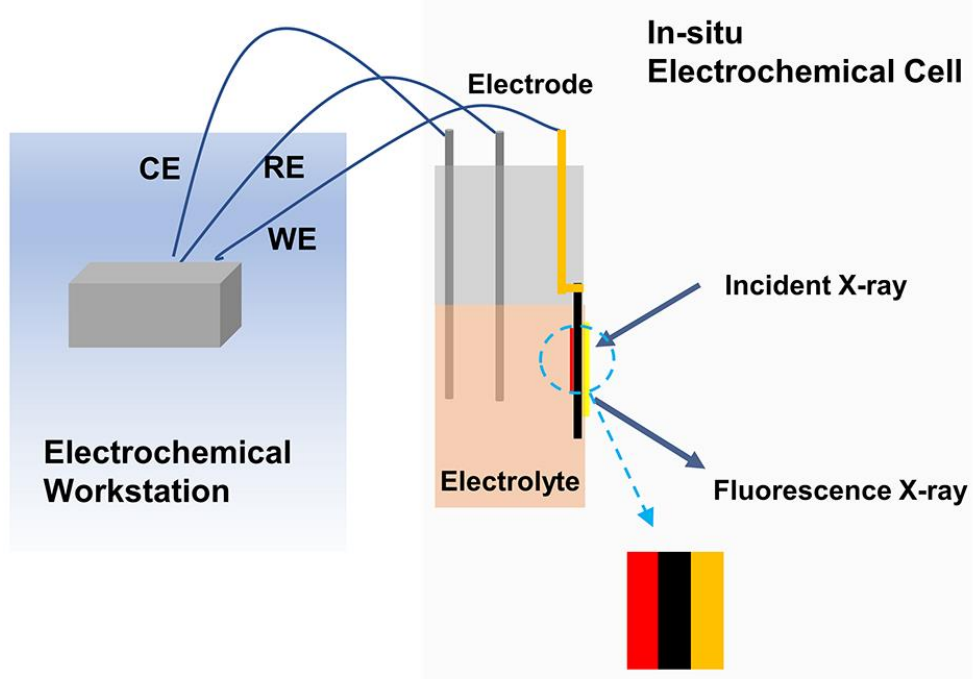

Catalyst, Carbon Paper, Kapton Membrane

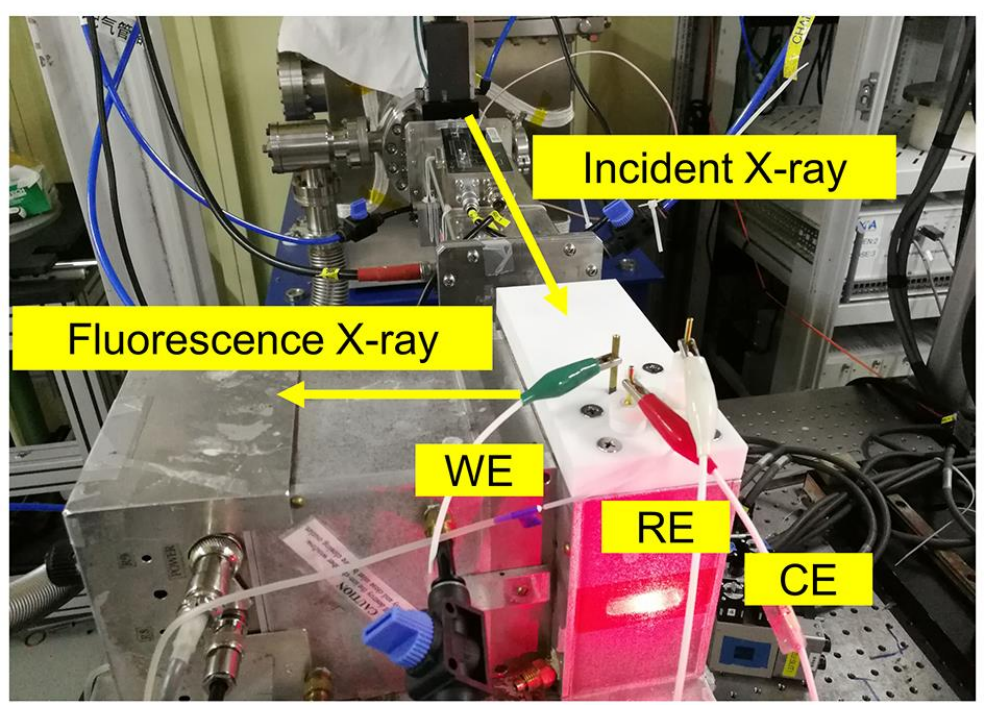

Figure S3. Equipment used for operando XAFS measurements. Schematical illustration of the equipment used for operando XAFS measurements (top). Digital photo taken during measurements (bottom). 


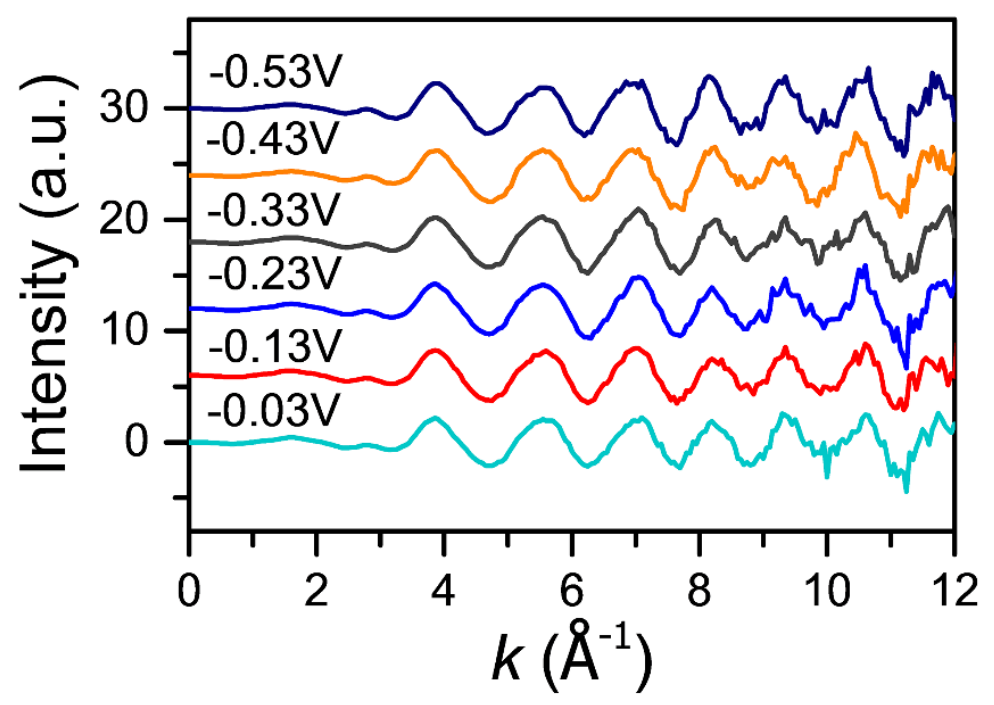

Figure S4. The raw $k^{2}$-weighted $k$-space spectra of SNM-Pt at different potentials. 

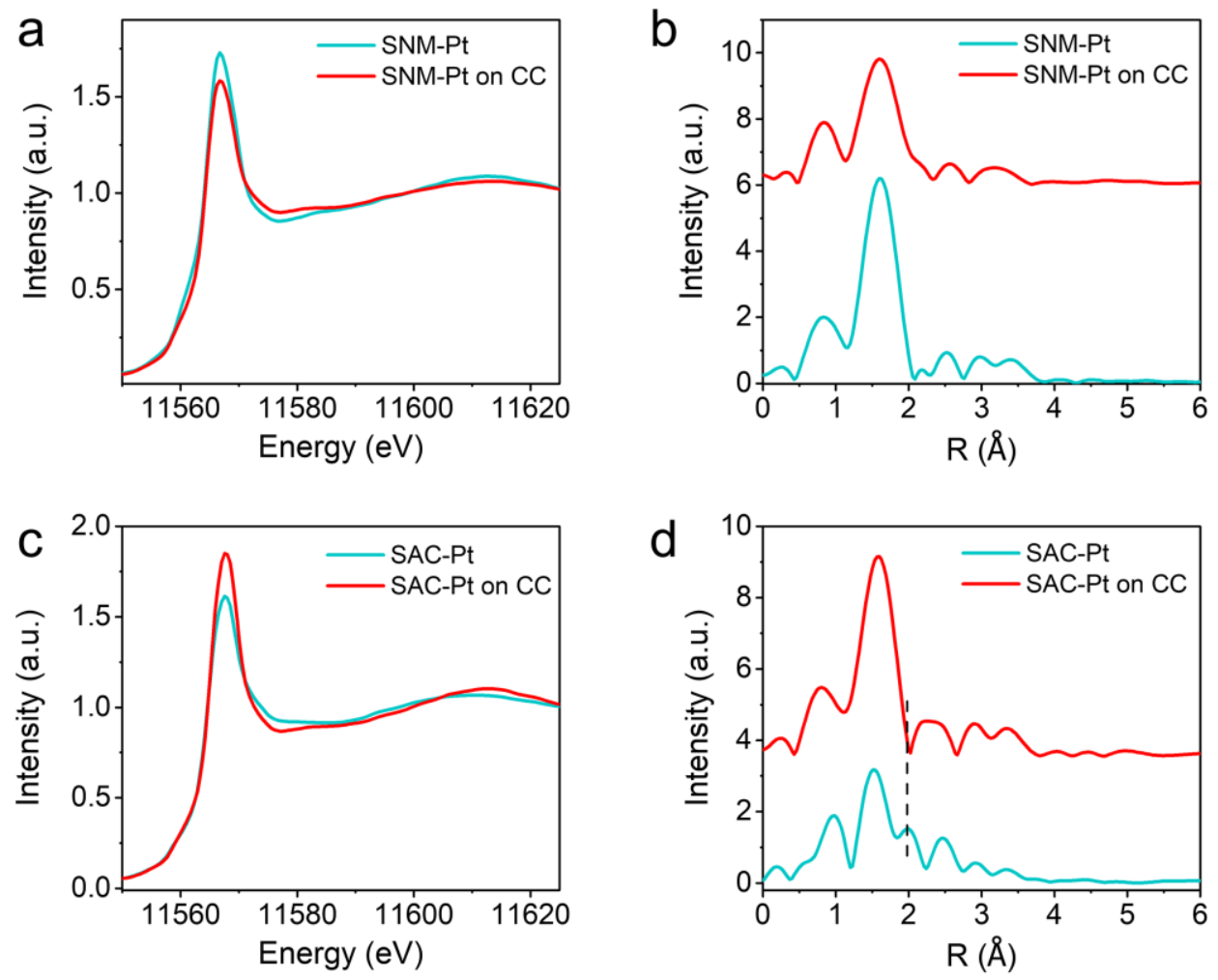

Figure S5. The XAS spectra evolution of (a, b) SNM-Pt and (c, d) SAC-Pt after the electrode preparation on CC. 

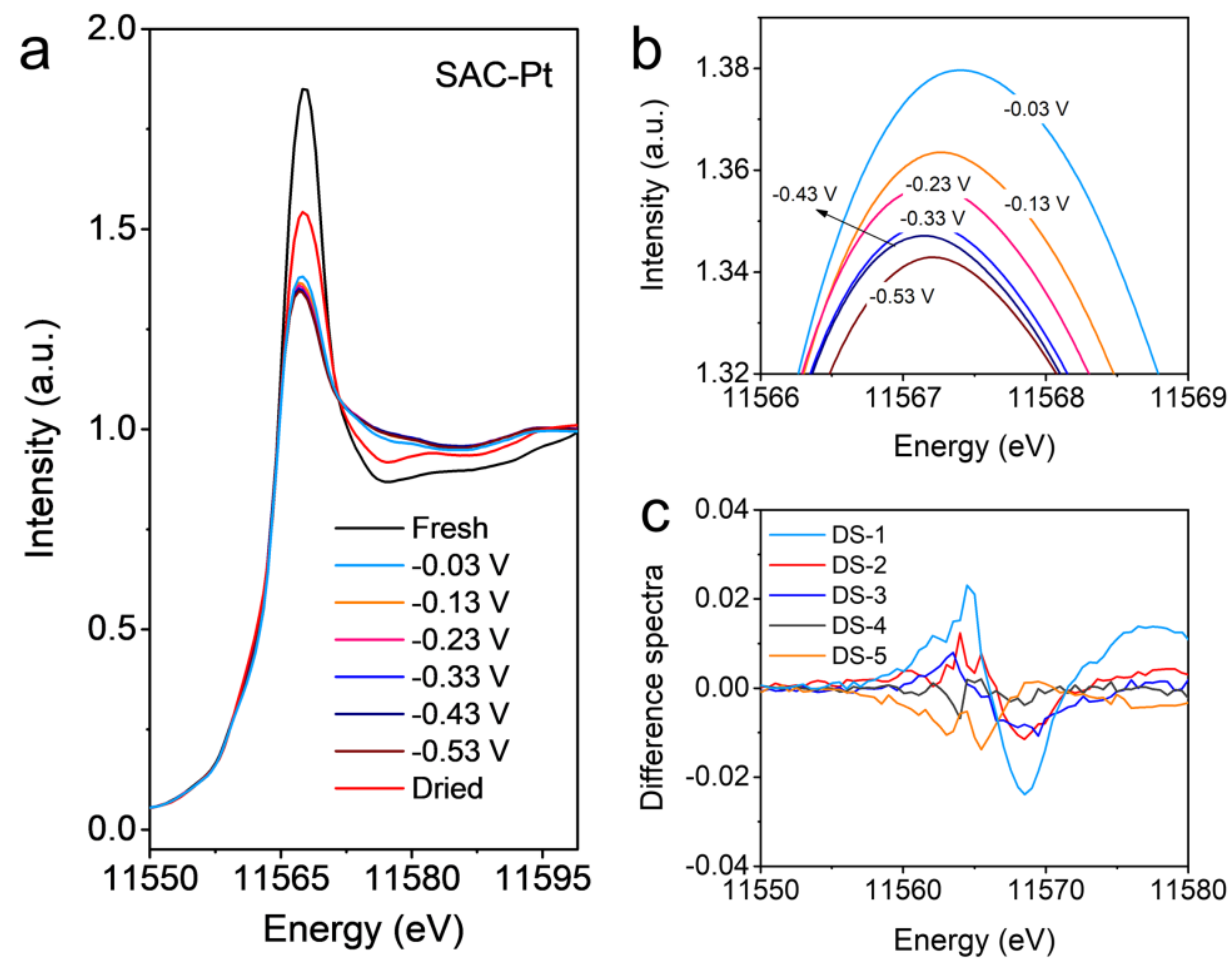

Figure S6. Operando XANES spectra of SAC-Pt at different potentials. 

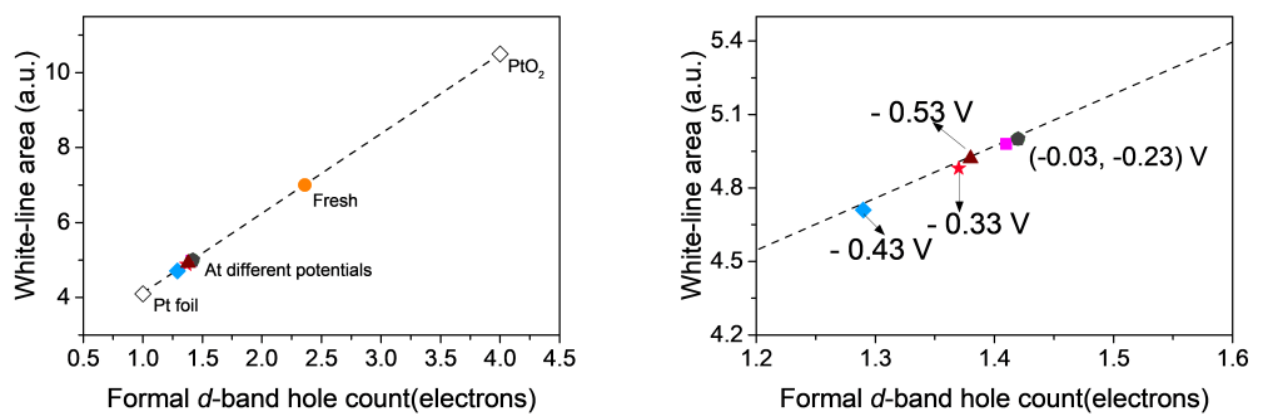

Figure S7. Linear relationship between the integrated white-line area and formal $5 d$ band hole count for $\mathrm{Pt}$ foil, $\mathrm{PtO}_{2}$, fresh $\mathrm{SNM}-\mathrm{Pt}$, and those at different potentials. XANES curve fitting analysis using a set of arctangent function and Lorentzian function in Athena program. 


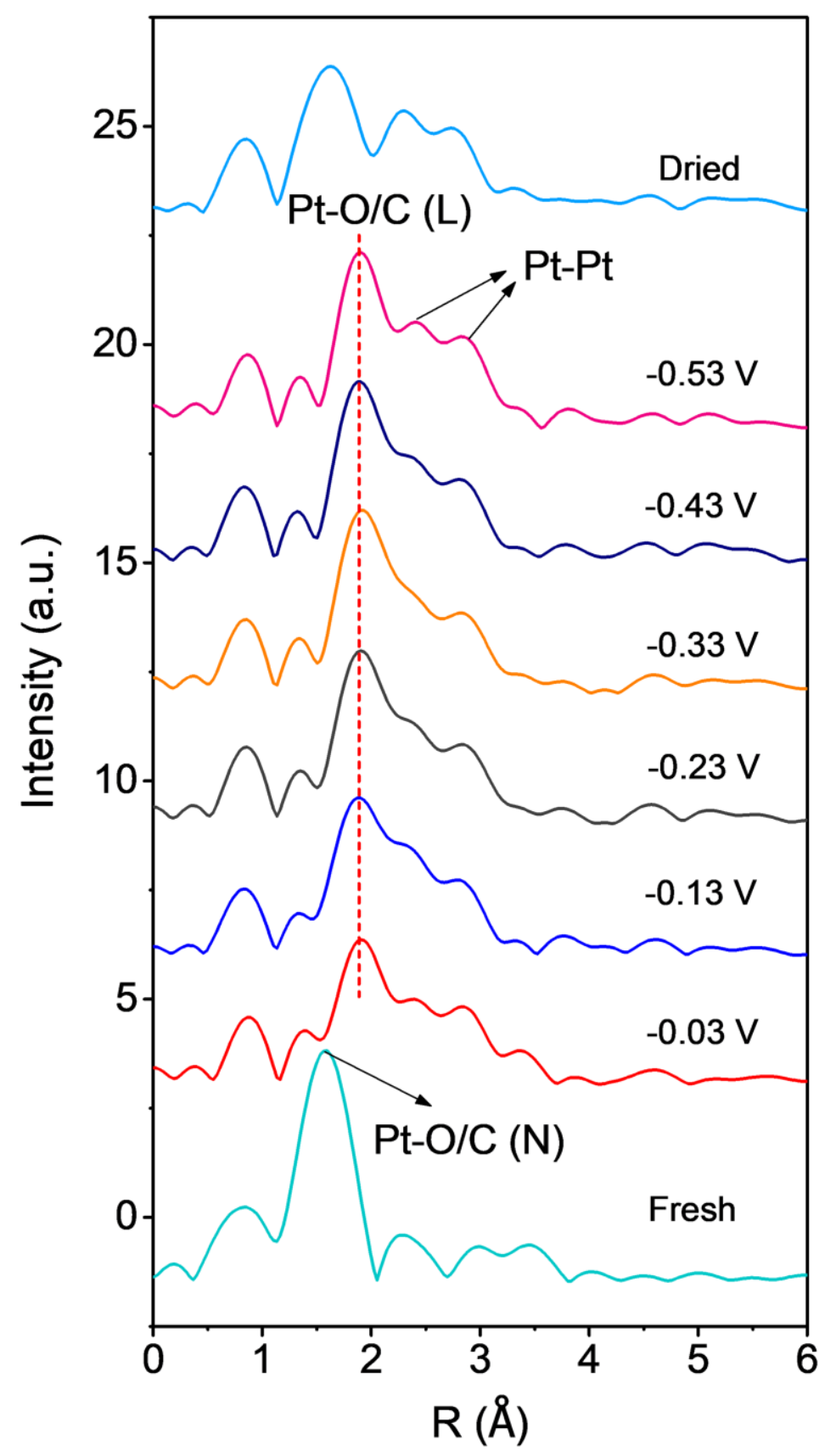

Figure S8. The operando FT-EXAFS spectra of SAC-Pt at different potentials. 

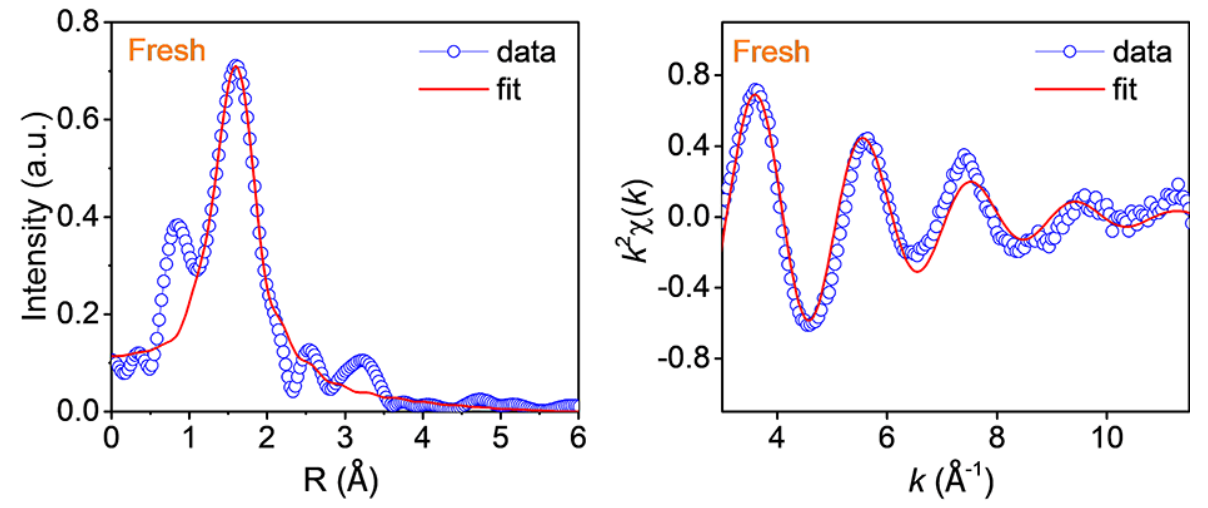

Figure S9. Experimental and fitted EXAFS spectra in R (left) and k (right) space for the fresh SNM-Pt loaded on CC. 

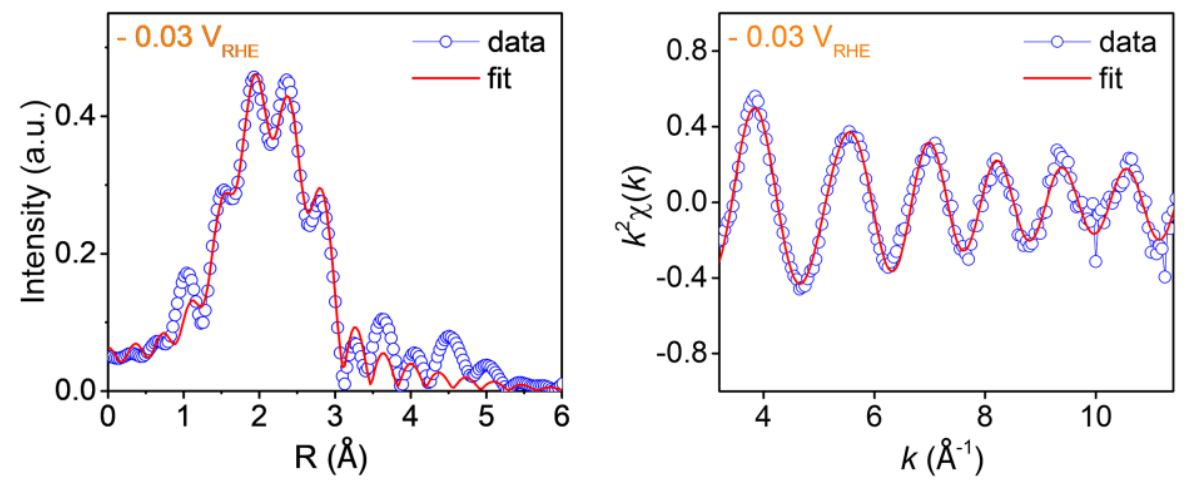

Figure S10. Experimental and fitted EXAFS spectra in R (left) and k (right) space for the SNM-Pt at potential of $-0.03 \mathrm{~V}_{\mathrm{RHE}}$. 

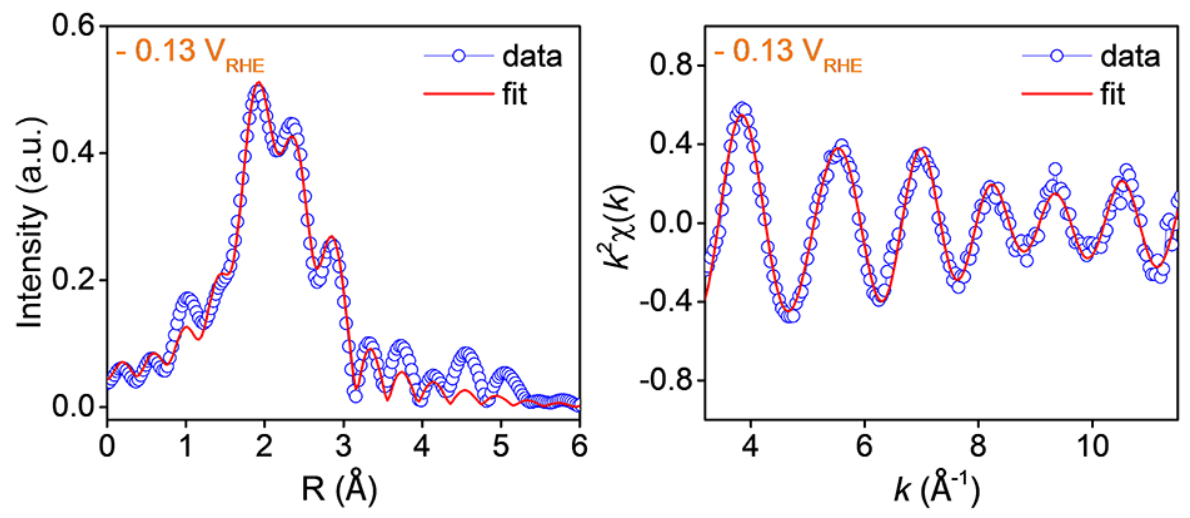

Figure S11. Experimental and fitted EXAFS spectra in R (left) and k (right) space for the SNM-Pt at potential of $-0.13 \mathrm{~V}_{\mathrm{RHE}}$. 

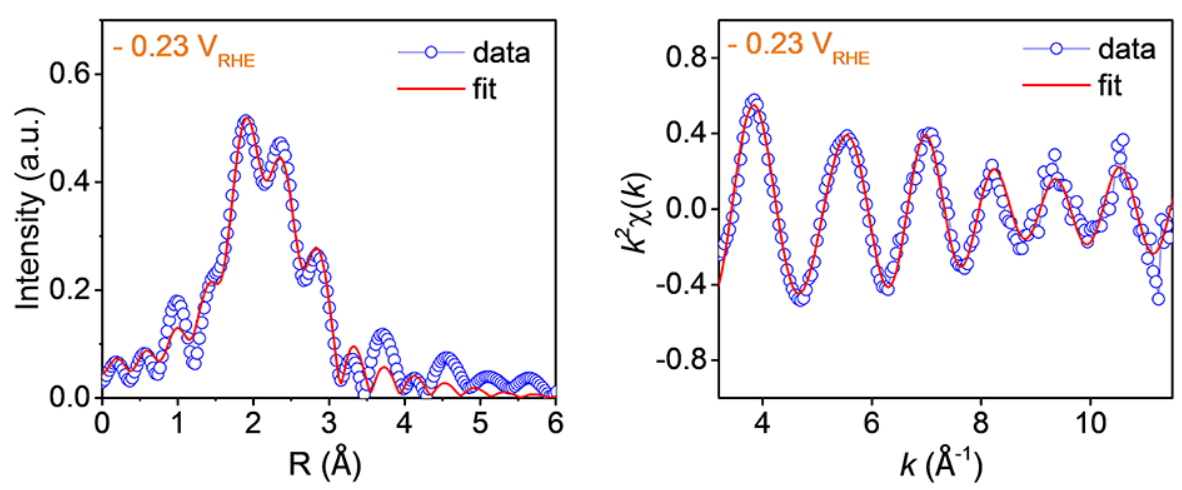

Figure S12. Experimental and fitted EXAFS spectra in R (left) and k (right) space for the SNM-Pt at potential of $-0.23 \mathrm{~V}_{\mathrm{RHE}}$. 

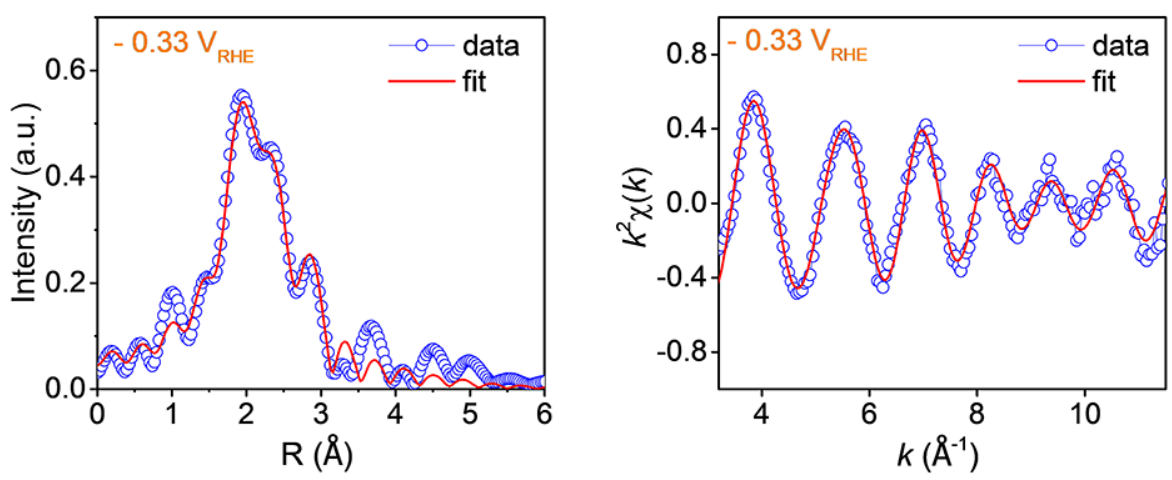

Figure S13. Experimental and fitted EXAFS spectra in R (left) and k (right) space for the SNM-Pt at potential of $-0.33 \mathrm{~V}_{\mathrm{RHE}}$. 

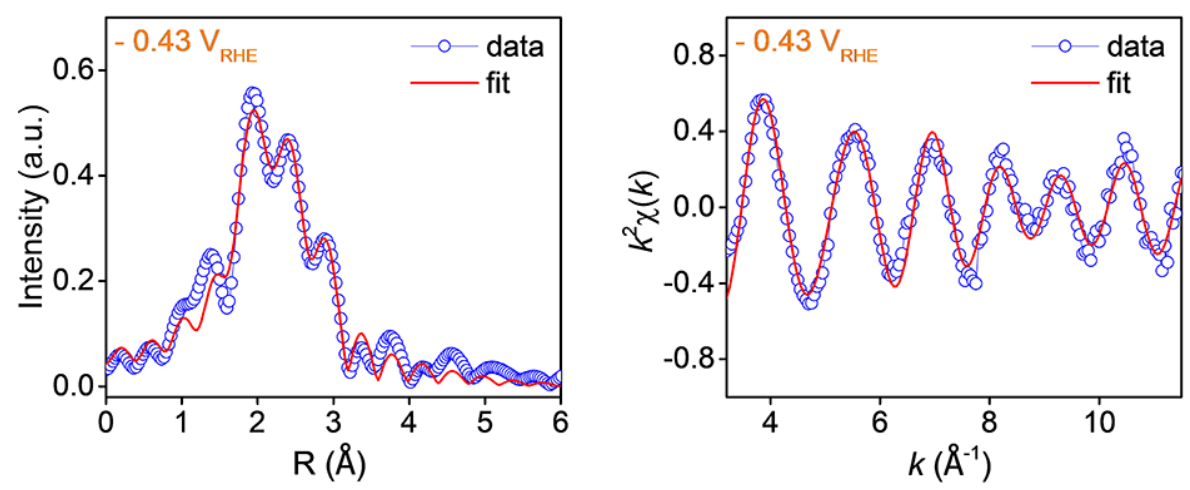

Figure S14. Experimental and fitted EXAFS spectra in R (left) and k (right) space for the SNM-Pt at potential of $-0.43 \mathrm{~V}_{\text {RHE}}$. 

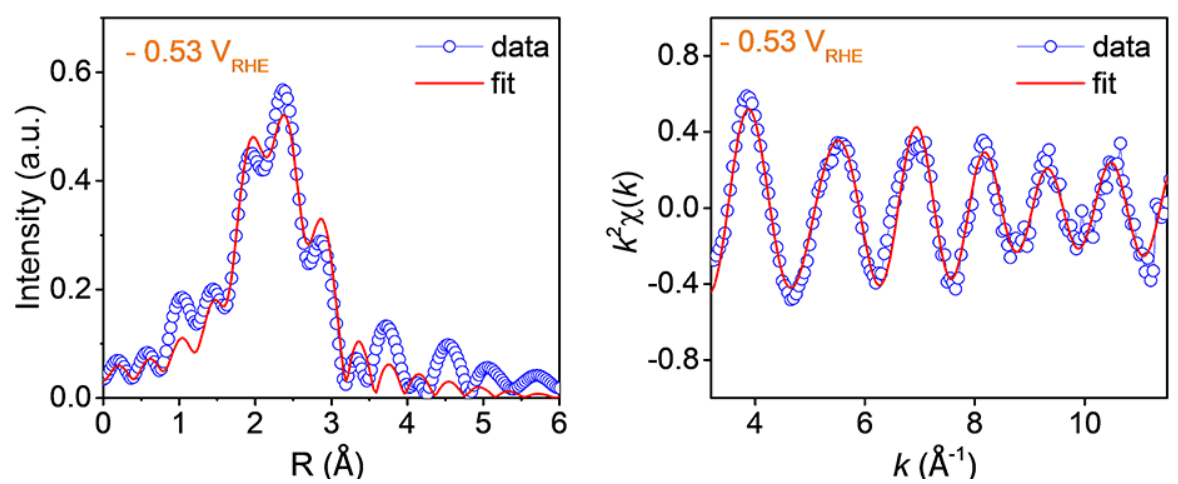

Figure S15. Experimental and fitted EXAFS spectra in R (left) and k (right) space for the SNM-Pt at potential of $-0.53 \mathrm{~V}_{\mathrm{RHE}}$. 

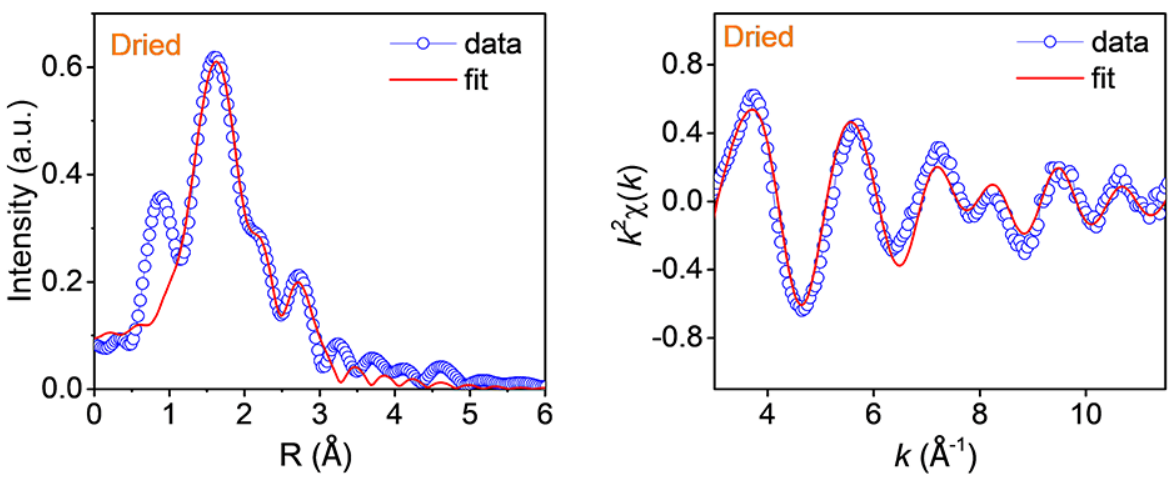

Figure S16. Experimental and fitted FT- EXAFS spectra in the R (left) and k (right) space for the dried SNM-Pt after operando measurements. 


\section{Supplementary Tables}

Table S1. Comparison of HER performance between SNM-Pt and the recently reported Pt-based electrocatalysts.

\begin{tabular}{|c|c|c|c|c|}
\hline Sample & $\begin{array}{l}\text { Overpotential } \\
\text { (@ } 10 \mathrm{~mA} \\
\left.\mathrm{~cm}^{-2}\right)\end{array}$ & $\begin{array}{l}\text { Mass activity } \\
\quad(@ 50 \mathrm{~m})\end{array}$ & $\begin{array}{c}\text { Tafel } \\
\text { Slope } \\
\text { (mV/dec) }\end{array}$ & Note \\
\hline SNM-Pt & 18.0 & 19.0 & 27 & This work \\
\hline $\mathrm{Pt}_{1} / \mathrm{OLC}$ & 38.0 & 13.2 & 35 & Nat. Energy. $2019^{1}$ \\
\hline $\mathrm{Pt}$ SAs/DG & 23.0 & 26.2 & 25 & J. Am. Chem. Soc. $2019^{2}$ \\
\hline $\mathrm{Pt}$ SASs/AG & 12.0 & 22.4 & 29.3 & Energy Environ. Sci. $2019^{3}$ \\
\hline $\mathrm{Mo}_{2} \mathrm{TiC}_{2} \mathrm{~T}_{\mathrm{x}}-\mathrm{PtsA}_{\mathrm{SA}}$ & 30.0 & 2.6 & 30 & Nat. Catal, $2018^{4}$ \\
\hline $\mathrm{Pt} \mathrm{SA} / \mathrm{m}-\mathrm{WO}_{3-\mathrm{x}}$ & 46.9 & 12.8 & 45 & Angew. Chem. $2019^{5}$ \\
\hline $\mathrm{Pt} / \mathrm{CNTs}-\mathrm{ECR}$ & 34 & - & 26 & J. Mater. Chem. A. $2019^{6}$ \\
\hline Pt-AC/DG-500 & 21 & - & 27.5 & J. Am. Chem. Soc. $2020^{7}$ \\
\hline $\mathrm{Pt}_{5} / \mathrm{HMCS}$ & 20.7 & - & 28.4 & Adv. Mater. $2019^{8}$ \\
\hline Ni-MOF@Pt & 43 & - & 30 & Nano. Lett. $2019^{9}$ \\
\hline Pt@PCM & 105 & - & 65.3 & Sci. Adv. $2018^{10}$ \\
\hline $\mathrm{AL}-\mathrm{Pt} / \mathrm{Pd}{ }_{3} \mathrm{~Pb}$ & 13.8 & - & 18 & J. Am. Chem. Soc. $2019^{11}$ \\
\hline $\mathrm{Pt}-\mathrm{WO}_{3}$ & 39 & - & 32.3 & Nano Energy. $2020^{12}$ \\
\hline Pt-GT-1 & 18 & - & 24 & Nat. Energy. $2018^{13}$ \\
\hline AC Pt-NG/C & 35.3 & - & 31 & ACS Catal. $2019^{14}$ \\
\hline $1 \% \mathrm{PtW}_{6} \mathrm{O}_{24} / \mathrm{C}$ & 22 & - & 29.8 & Nat. Commun. $2020^{15}$ \\
\hline $\mathrm{PtNC} / \mathrm{S}-\mathrm{C}$ & 11 & - & 23.5 & Nat. Commun. $2019^{16}$ \\
\hline $\mathrm{Pt}_{1} / \mathrm{N}-\mathrm{C}$ & 19 & - & 14.2 & Nat. Commun. $2020^{17}$ \\
\hline Vo-rich $\mathrm{Pt} \mathrm{TiO} 2$ & - & 19.51@70mV & 34.0 & Angew. Chem. $2021^{18}$ \\
\hline
\end{tabular}




\begin{tabular}{cccc}
$\mathrm{PtSe}_{2} / \mathrm{Pt}$ & 42 & 53.0 & Angew. Chem. $2021^{19}$ \\
$\mathrm{PtTe}_{2}-600$ & 22 & 29.9 & Nat. Commun. $2021^{20}$ \\
$(\mathrm{Ru}-\mathrm{N}) @ \mathrm{Pt}$ & 15 & 25.0 & J. Mater. Chem. A. $2021^{21}$ \\
$\mathrm{Pt} @ \mathrm{MoS}_{2}$ & 88.4 & 55.7 & Nano Energy. $2021^{22}$ \\
\hline
\end{tabular}


Table S2. EXAFS fitting parameters of SNM-Pt under different states.

\begin{tabular}{|c|c|c|c|c|c|c|}
\hline Sample & Path & $\mathbf{C N}$ & $\mathbf{R}(\mathbf{A})$ & $\Delta E_{0}(e V)$ & $\sigma^{2}\left(10^{-3} \AA^{2}\right)$ & $\mathbf{R}$ factor \\
\hline Fresh & $\mathrm{Pt}-\mathrm{O}(\mathrm{N})$ & $3.7 \pm 0.1$ & $2.01 \pm 0.02$ & $9.5 \pm 1.8$ & $9.8 \pm 2.8$ & 0.010 \\
\hline \multirow[t]{3}{*}{$-0.03 V_{\text {RHE }}$} & $\mathrm{Pt}-\mathrm{O}(\mathrm{N})$ & $0.5 \pm 0.1$ & $2.00 \pm 0.03$ & $10.5 \pm 3.1$ & $7.0^{*}$ & 0.005 \\
\hline & $\mathrm{Pt}-\mathrm{O}(\mathrm{L})$ & $2.4 \pm 0.1$ & $2.47 \pm 0.01$ & $-3.7 \pm 1.1$ & $7.0^{*}$ & \\
\hline & Pt-Pt & $4.1 \pm 0.1$ & $2.71 \pm 0.01$ & $-1.8 \pm 0.7$ & $7.0^{*}$ & \\
\hline \multirow[t]{2}{*}{$-0.13 V_{\text {RHE }}$} & $\mathrm{Pt}-\mathrm{O}(\mathrm{L})$ & $3.6 \pm 0.1$ & $2.45 \pm 0.01$ & $-7.8 \pm 1.0$ & $7.0^{*}$ & 0.006 \\
\hline & $\mathrm{Pt}-\mathrm{Pt}$ & $4.1 \pm 0.2$ & $2.72 \pm 0.01$ & $-1.5 \pm 1.0$ & $7.0^{*}$ & \\
\hline \multirow[t]{2}{*}{$-0.23 V_{\text {RHE }}$} & $\mathrm{Pt}-\mathrm{O}(\mathrm{L})$ & $3.7 \pm 0.2$ & $2.45 \pm 0.01$ & $-7.8 \pm 1.4$ & $7.0^{*}$ & 0.010 \\
\hline & Pt-Pt & $4.3 \pm 0.2$ & $2.71 \pm 0.01$ & $-2.8 \pm 1.3$ & $7.0^{*}$ & \\
\hline \multirow[t]{2}{*}{$-0.33 V_{\text {RHE }}$} & $\mathrm{Pt}-\mathrm{O}(\mathrm{L})$ & $3.9 \pm 0.1$ & $2.48 \pm 0.01$ & $-5.4 \pm 0.8$ & $7.0^{*}$ & 0.004 \\
\hline & Pt-Pt & $3.9 \pm 0.1$ & $2.71 \pm 0.01$ & $-2.7 \pm 0.9$ & $7.0^{*}$ & \\
\hline \multirow[t]{2}{*}{$-0.43 V_{\text {RHE }}$} & $\mathrm{Pt}-\mathrm{O}(\mathrm{L})$ & $3.8 \pm 0.3$ & $2.48 \pm 0.02$ & $-4.3 \pm 1.7$ & $7.0^{*}$ & 0.016 \\
\hline & Pt-Pt & $4.6 \pm 0.3$ & $2.74 \pm 0.01$ & $-1.1 \pm 1.7$ & $7.0^{*}$ & \\
\hline \multirow[t]{2}{*}{$-0.53 V_{\text {RHE }}$} & $\mathrm{Pt}-\mathrm{O}(\mathrm{L})$ & $2.9 \pm 0.2$ & $2.49 \pm 0.01$ & $-2.2 \pm 1.8$ & $7.0^{*}$ & 0.015 \\
\hline & Pt-Pt & $5.2 \pm 0.3$ & $2.74 \pm 0.01$ & $0.3 \pm 0.5$ & $7.0^{*}$ & \\
\hline \multirow[t]{2}{*}{ Dried } & $\mathrm{Pt}-\mathrm{O}(\mathrm{N})$ & $3.2 \pm 0.3$ & $2.00 \pm 0.01$ & $9.5 \pm 1.1$ & $10.5 \pm 1.9$ & 0.014 \\
\hline & Pt-Pt & $2.5 \pm 0.3$ & $2.71 \pm 0.01$ & $8.6 \pm 1.9$ & $7.7 \pm 2.1$ & \\
\hline
\end{tabular}

$\overline{\mathrm{CN}}$, coordination number; $\mathrm{R}$, distance between absorber and backscatter atoms; $\sigma^{2}$, Debye-Waller factor; $\Delta \mathrm{E}_{0}$, inner potential correction; R-factor, indicative of fitting goodness; the fixed values $(*)$ in the fitting process. 
Table S3. The average Pt-Pt CN calculated from the optimized models. (Only the PtPt with a radial distance between 2.45-3.00 $\AA$ is included)

\begin{tabular}{|c|c|c|c|}
\hline & $\mathrm{Pt}_{13} \mathrm{H}_{0}$ & $\mathrm{Pt}_{13} \mathrm{H}_{6}$ & $\mathrm{Pt}_{13} \mathrm{H}_{20}$ \\
\hline Pt1 & 6 & 6 & 6 \\
\hline Pt2 & 4 & 4 & 3 \\
\hline Pt3 & 4 & 4 & 4 \\
\hline Pt4 & 4 & 4 & 4 \\
\hline Pt5 & 6 & 6 & 8 \\
\hline Pt6 & 4 & 4 & 3 \\
\hline Pt7 & 3 & 3 & 5 \\
\hline Pt8 & 4 & 4 & 5 \\
\hline Pt9 & 3 & 3 & 3 \\
\hline Pt10 & 6 & 6 & 7 \\
\hline Pt11 & 6 & 6 & 5 \\
\hline Pt12 & 6 & 6 & 5 \\
\hline Pt13 & 4 & 4 & 4 \\
\hline Average & 4.3 & 4.6 & 4.8 \\
\hline
\end{tabular}




\section{References}

1. Liu, D.; Li,X.; Chen, S.; Yan, H.; Wang, C.; Wu,C.; Haleem, Y. A.; Duan, S.; Lu, J.; Ge, B., Atomically dispersed platinum supported on curved carbon supports for efficient el ectrocatalytic hydrogen evolution. Nat. Energy 2019, 4 (6), 512-518.

2. Qu, Y.; Chen, B.; Li, Z.; Duan, X.; Wang, L.; Lin, Y.; Yuan, T.; Zhou, F.; Hu, Y.; Yang, Z.; Zhao, C.; Wang, J.; Zhao, C.; Hu, Y.; Wu, G.; Zhang, Q.; Xu, Q.; Liu, B.; Gao, P.; You, R.; Huang, W.; Zheng, L.; Gu, L.; $W u, Y$.; Li, Y., Thermal Emitting Strategy to Synthesize Atomically Dis persed Pt Metal Sites from Bulk Pt Metal. J. Am. Chem. Soc. 2019, 141 (11), 4505-4509.

3. Ye, S.; Luo, F.; Zhang, Q.; Zhang, P.; Xu, T.; Wang, Q.; He, D.; Guo, L.; Zhang, Y.; He, C., Highly stable single Pt atomic sites anchored on a niline-stacked graphene for hydrogen evolution reaction. Energy Environ. Sci. 2019, 12 (3), 1000-1007.

4. Zhang, J.; Zhao, Y.; Guo, X.; Chen, C.; Dong, C.-L.; Liu, R.-S.; Han, C.-P.; Li,Y.; Gogotsi, Y.; Wang, G., Single platinum atoms immobilized on an MXene as an efficient ca talyst for the hydrogen evolution reaction. Nat. Catal. 2018, 1 (12), 985-992.

5. Park, J.; Lee, S.; Kim, H. E.; Cho, A.; Kim, S.; Ye, Y.; Han, J. W.; Lee, H.; Jang, J. H.; Lee, J., Investigation of the Support Effect in Atomically Dispersed Pt on WO3-x for Utilization of Pt in the Hydrogen Evolution Reaction. Angew. Chem. Int. Ed. 2019, 58 (45), 16038-16042.

6. Bao, X.; Gong, Y.; Chen, Y.; Zhang, H.; Wang, Z.; Mao, S.; Xie, L.; Jiang, Z.; Wang, Y., Carbon vacancy defect-activated Pt cluster for hydrogen generation. J. Mater. Chem. A. 2019, 7 (25), 15364-15370.

7. Cheng, Q.; Hu, C.; Wang, G.; Zou, Z.; Yang, H.; Dai, L., Carbon-Defect-Driven Electroless Deposition of Pt Atomic Clusters for Highly Efficient Hydrogen Evolution. J. Am. Chem. Soc. 2020, 142 (12), 5594-5601.

8. Wan, X. K.; Wu, H. B.; Guan, B. Y.; Luan, D.; Lou, X. W. D., Confining Sub-Nanometer Pt Clusters in Hollow Mesoporous Carbon Spheres for Boosting Hydrogen Evolution Activity. Adv. Mater. 2020, 32 (7), e1901349.

9. Rui, K.; Zhao, G.; Lao, M.; Cui, P.; Zheng,X.; Zheng,X.; Zhu, J.; Huang, W.; Dou, S.X.; Sun, W., Direct Hybridization of Noble Metal Nanostructures on 2D Metal-Organic Framework Nanos heets To Ca talyze Hydrogen Evolution. Nano Lett. 2019, 19(12), 8447-8453.

10. Zhang, H.; An, P.; Zhou, W.; Guan, B.Y.; Zhang, P.; Dong, J.; Lou, X. W. D., Dynamic traction of lattice-confined platinum atoms into mesoporous carbon matrix for hydrogen evolution reaction. Sai. Adv. 2018, 4 (1), ea ao6657.

11. Yao,Y.; Gu,X.-K.; He, D.; Li,Z.; Liu,W.; Xu, Q.; Yao,T.; Lin, Y.; Wang, H.-J.; Zhao, C.; Wang, X.; Yin, P.; Li, H.; Hong, X.; Wei, S.; Li, W.; Li, Y.; Wu, Y., Engineering Electronic Structure of Sub-monolayer Pt on Intermetallic Pd3Pb via Charge Transfer Boosts Hydrogen Evolution Rea ction. J. Am. Chem. Soc. 2019, 141 (51), 19964-19968.

12. Xie, C.; Chen, W.; Du, S.; Yan, D.; Zhang, Y.; Chen, J.; Liu, B.; Wang, S., In-situ phase transition of WO3 boosting electron and hydrogen transfer for enhancing hydrogen evolution on Pt. Nano Energy 2020, 104653.

13. Tiwari, J.N.; Sultan, S.; Myung, C. W.; Yoon, T.; Li, N.; Ha, M.; Harzandi, A. M.; Park, H. J.; Kim, D. Y.; Chandrasekaran, S. S., Mul ticomponent el ectrocatalyst with ultralow Pt loading and high hydrogen evolution activity. Nat. Energy 2018, 3 (9), 773-782.

14. Sun, M.; Ji, J.; Hu, M.; Weng, M.; Zhang, Y.; Yu, H.; Tang, J.; Zheng, J.; Jiang, Z.; Pan, F., Overwhelming the Performance of Single Atoms with Atomic Clusters for Platinum-Catalyzed 
Hydrogen Evolution. ACS Catal. 2019, 9 (9), 8213-8223.

15. Yu, F.Y.; Lang, Z. L.; Yin, L.Y.; Feng, K.; Xia,Y.J.; Tan, H. Q.; Zhu, H.T.; Zhong, J.; Kang, Z. H.; Li, Y. G., Pt-O bond as a nactive site superior to $\mathrm{Pt}(0)$ in hydrogen evolution reaction. Nat. Commun. 2020,11(1), 490.

16. Yan, Q.-Q.; Wu, D.-X.; Chu, S.-Q.; Chen,Z.-Q.; Lin, Y.; Chen, M.-X.; Zhang, J.; Wu,X.-J.; Liang, H.-W., Reversing the charge transfer between platinum and sulfur-doped carbon support for el ectrocatalytic hydrogen evolution. Nat. commun. 2019, 10 (1), 1-9.

17. Fang, S.; Zhu,X.; Liu,X.; Gu,J.; Liu,W.; Wang, D.; Zhang,W.; Lin, Y.; Lu, J.; Wei, S.; $\mathrm{Li}, \mathrm{Y}$; $Y$ Yao, T., Uncovering near-free platinum single-atom dynamics during el ectrochemical hydrogen evolution reaction. Nat. Commun. 2020, 11 (1), 1029.

18. Wei, Z. W.; Wang, H. J.; Zhang, C.; Xu, K.; Lu, X. L.; Lu, T. B., Reversed Charge Transfer and Enhanced Hydrogen Spillover in Platinum Nanoclusters Anchored on Ti tanium Oxide with Rich Oxygen Vacancies Boost Hydrogen Evolution Reaction. Angew. Chem. Int.,2021, 60 (30), 16622-16627.

19. Wang, Z.; Xiao, B.; Lin, Z.; Xu, Y.; Lin,Y.; Meng, F.; Zhang, Q.; Gu, L.; Fang, B.; Guo, S.; Zhong, W., $\mathrm{PtS} \mathrm{e}_{2} / \mathrm{Pt}$ heterointerface with reduced coordination for boosted hydrogen evolution reaction. Angew. Chem. Int. 2021, doi: 10.1002/ange.202110335.

20. Li, X.; Fang, Y.; Wang, J.; Fang, H.; Xi, S.; Zhao, X.; Xu, D.; Xu, H.; Yu, W.; Hai, X.; Chen, C.; Yao, C.; Tao, H. B.; Howe, A. G. R.; Pennycook, S. J.; Liu, B.; Lu, J.; Su, C., Ordered cl ustering of single atomic Tevacancies in atomically thin $\mathrm{PtTe}_{2}$ promotes hydrogen evolution catalysis. Nat. Commun. 2021, 12(1), 2351.

21. Luo, M., Cai, J. Y., Zou, J. S., Jiang, Z., Wang, G. M., Kang, X. W., Promoted Alkaline hydrogen evolution by an N-doped Pt-Ru Single Atom Al loy. J. Mater. Chem. A, 2021, 9, 14941-14947.

22. Li, Y., Gu, Q. F., Johannessen, B., Zheng, Z., Li, C., Luo, Y. T., Zhang, Z. Y., Zhang, Q., Fan, H. N., Luo, W. B., Liu, B. L., Dou, S. X., Li U, H. K., Synergistic Pt Doping and Phase Conversion Engineering in Twodimensional MoS2 for efficient hydrogen evolution. Nano Energy, 2021, 84, 105898. 